\title{
« Dans les marges du cahier de textes ». Quelques aspects de l'autoréflexivité dans l'écriture guibertienne
}

\author{
Alessandro Badin \\ Université de Vérone
}

« Tchekhov est un petit con, il prétend qu'il écrit ses nouvelles dans sa baignoire. ». Le narrateur-écrivain de L'Incognito fait cette remarque à propos de l'écrivain russe (Guibert, 1989a, p. 63) et il réfléchit sur cette insolite habitude de travail alors qu'il est lui-même en train de prendre un bain, petit plaisir inventé pour contraster avec l'austérité de la Villa Médicis à Rome, où il est censé écrire l'histoire de sa vie. L'attention portée à un aspect aussi banal, apparemment insignifiant du travail de Tchekhov, fait partie d'une multitude de descriptions, de réflexions, d'évocations plus ou moins élaborées concernant 
le travail de l'auteur, nous ouvrant une fenêtre sur son statut, mais aussi sur son mode de vie, sur ses habitudes. Faisant de lui-même le personnage principal de son œuvre, Guibert nous invite nécessairement à découvrir son monde, son œuvre, son laboratoire d'écriture. Corporelle, photographique, sidéenne, introspective ou penchée vers la recherche de l'autre, cette écriture se met parfois en quête d'elle-même, de son statut, de sa genèse, de sa matérialité.

L'immédiateté de la photographie, partie intégrante de l'œuvre de Guibert, nous permet d'appréhender plus facilement l'insertion, à côté de portraits, d'objets familiers ou singuliers, des outils de son propre travail d'écrivain, tels les instruments (stylo ou machine à écrire), les supports (feuilles, carnets, etc.), les résultats d'écriture (manuscrits ou feuilles dactylographiées); de plus, les photos nous montrent les cadres de la création, nous font franchir les portes de l'atelier au sens propre du terme : bibliothèque, bureau, table de travail. Comme l'observe Robert Pujade dans son livre sur Guibert photographe, une partie des images est «consacrée aux autoportraits et à ce qu'on pourrait appeler des extensions de l'autoportrait: les objets familiers, les lieux et les instruments de travail» (p. 71). À propos des objets qui apparaissent dans ces autoportraits, il ajoute que «les objets les plus récurrents sont ceux qui sont associés à la table de travail de l'écrivain : la machine à écrire héritée du grand oncle paternel, entourée du stylo Mont Blanc, de la bouteille d'encre Pelikan et des feuilles de brouillon ordonnées » (p. 83). Ces éléments se situent donc au centre de la représentation de soi, de la projection de sa propre identité. 
Cohérent avec son projet de dévoilement jusqu'au paroxysme, Guibert développe toutefois une attitude sinon ambiguë, du moins ambivalente par rapport à son métier d'écrivain: constamment exposée, montrée, l'écriture est également cachée, voilée, comme protégée par l'écrivain, poussé par une sorte de pudeur, de réserve, vers l'œil indiscret de l'observateur qui force les limites de l'espace créateur. Mais l'attitude de Guibert est double, et s'il ne cesse de manifester, de dénoncer la peur d'être espionné ; il trace néanmoins le portrait de lui-même en écrivain, composant sous nos yeux une mosaïque plutôt exhaustive de ses habitudes de travail, de son laboratoire. Et la silhouette qu'il trace est celle d'un écrivain tout à fait « polymorphe » (Boulé, p. 12), qui programme parfois ses séances d'écriture en choisissant les lieux, les outils, le calme idéal, ou qui, d'autres fois, improvise, poussé par l'urgence de fixer une impression, un détail, une circonstance : tout support, tout lieu devient apte à abriter la création littéraire.

Par le fait même d'évoquer son statut d'écrivain et de parsemer ses récits d'allusions à ses expériences d'écriture, Guibert indique au lecteur le chemin pour une réflexion sur le statut fictionnel du récit, lui dévoilant la naissance de l'artifice littéraire : par des dispositifs de représentation de l'écriture en train de se faire l'auteur devient, donc, autoréflexif.

«La notion même d'autoréflexivité peut prêter à discussion» (Herman, p.1) ainsi que le complexe réseau terminologique associé à l'étiquette générale de "phénomènes de spécularité ». Une constellation de termes constituent autant de déclinaisons pour désigner ces moments dans un texte où la littérature, l'écriture «se met en scène, [...] se commente, se 
regarde et se démultiplie » (Wessler, p. 18). Tout particulièrement en ce qui concerne le terme "réflexivité", on désigne la "tendance d'un texte à revenir sur lui-même, à se représenter, ou à se remettre en question en tant que tel » (p. 19). Ce terme, issu de la spéculation philosophique, a connu un glissement de sens, ne désignant plus seulement la faculté de la conscience à l'analyse de sa propre activité, mais aussi la capacité de l'écriture et de la littérature de revenir sur ellesmêmes, de se réfléchir, de devenir les instruments de leur propre miroitement et de leur propre analyse. La littérature dans sa globalité, de la même façon que l'œuvre de tel ou tel auteur en action, semble se miroiter, s'observer, s'autoanalyser: dans les images spéculaires ainsi formées «se dessine quelque chose d'autre que leur propre reflet, et qui leur donne un sens » (p. 12).

Désignant une activité purement intellectuelle, autoréflexivité a été employé d'abord par la critique d'art, l'empruntant à son tour à l'anthropologie. Le terme a connu un glissement ultérieur vers la critique littéraire au cours des années 1980 (Wessler, p. 25); dans ce cadre, le terme finit par indiquer un texte « qui thématise (notamment dans l'univers de sa fiction) l'un de ses ingrédients constitutifs, qu'il s'agisse de son langage, de sa structure, de sa fonction, de sa genèse » (p. 25).

En outre, il y a un nombre important de procédés métatextuels qui peuvent être considérés comme autant d'occasions autoréflexives. Symboles de l'écriture ou de la lecture, «formes métatextuelles iconiques » (Lepaludier, p. 32) et tout ce qui mène à un raisonnement sur le caractère artificiel de la narration et de la communication littéraire peuvent être 
considérés comme autant d'indices de réflexivité. Dans ce sens, l'intertextualité aussi en fait partie puisque, pendant la lecture d'un texte, "le recours à un autre texte provoque chez le lecteur une interrogation sur la nature du texte lu, une perception de son artifice, de sa littérarité » (p. 33). En général, et cela est particulièrement pertinent dans le cas de Guibert, la notion d'intertextualité devrait être élargie aux autres modes d'expression artistique et culturelle, aux autres domaines de l'art et de la création, à partir du moment où « les textes [qui] font allusion à des peintures, des sculptures, des styles d'architecture, des affiches, etc., déclench[e]nt une réflexion d'ensemble sur l'esthétique ou la culture » (p. 33). Pour l'œuvre de Guibert, cette dernière remarque est d'autant plus appropriée en raison des nombreuses figures d'écrivains et d'artistes qui peuplent ses récits, résultant de la transposition (auto)fictionnelle du milieu réellement fréquenté par l'auteur. Cette volonté de créer un pont, un véritable «dialogue scripturaire avec les auteurs qu'il admire » (Genon, p. 142) est le symptôme d'un plus grand désir de trouver sa voie entre tradition littéraire et recherche originale; voilà pourquoi « les procédés intertextuels mis en jeu sont révélateurs d'une quête d'identité scripturaire » (p. 148) constante, presque frénétique.

Dans le cadre de cette étude, nous nous intéresserons en particulier aux manifestations autoréflexives pour ainsi dire «au premier degré»: celles où l'œuvre s'exhibe elle-même, exhibe ses composantes matérielles, sa genèse. En effet, cette démarche métatextuelle opère à plusieurs niveaux dans l'œuvre de Guibert et pourrait être considérée comme l'une des approches privilégiées pour sa compréhension. À un niveau pour ainsi dire macroscopique, on peut retracer un réseau inter- et intratextuel complexe. À un niveau inférieur, plus 
localisé, nombreux sont les moments où la genèse de l'œuvre est décrite, évoquée, raisonnée. Si la dimension autoréflexive du roman À l'ami qui ne m'a pas sauvé la vie a déjà été soulignée, surtout en raison des "nombreuses allusions à sa genèse, à sa rédaction et même à l'inachèvement de l'histoire qu'il raconte » (Sarkonak, p. 171), il est important de mettre l'accent sur la présence de cette dimension dans l'ensemble de l'œuvre: les écrits littéraires mais aussi la photographie et le long métrage La Pudeur ou l'Impudeur.

\section{Un écrivain en quête de son statut}

Tout d'abord, Guibert nous rappelle qu'il est écrivain, évoquant constamment son statut, le voyant confirmé par les autres ou allant chercher lui-même cette confirmation, comme s'il recherchait sa propre image renvoyée par un miroir. Le souvenir des premières tentatives d'écriture littéraire, qui jaillit lors de la découverte de vieux manuscrits oubliés, est accompagné de la prétentieuse constatation de la valeur littéraire de ses écrits et de la certitude qu'un jour, il sera un grand écrivain : « [j]'ai toujours su que je serai un jour un grand écrivain, Jules n'y croyait pas, il se moquait de moi quand je lui disais que ces textes de jeunesse que tous les éditeurs refusaient seraient publiés un jour » (1991a, p.147); ou encore : « [j]'ai toujours su que je ferais un jour un grand succès d'un de mes livres, et qu'il ferait connaître tous les autres, mais David n'y croyait pas et se moquait de moi lui aussi » (1991a, p. 148). Ce statut lui est officiellement conféré lors de son admission en tant que pensionnaire à la Villa Médicis à Rome, institution culturelle qu'on peut facilement reconnaître dans l'«Académie espagnole» où se déroulent les singulières 
aventures constituant le récit de L'Incognito (voir Guibert, 1989a, p. 93). L'activité d'écrivain est définie explicitement comme une véritable profession (voir 1992a, p.38), dont Guibert perçoit les risques et la précarité auxquels tout métier est sujet (voir p. 25); le statut de cette activité est constamment questionné par l'auteur: il va chercher ses livres chez les libraires (voir 1989a, p. 83) et il n'est d'ailleurs pas indifférent aux implications éditoriales et commerciales de l'affaire, décrivant les rencontres et les négociations avec les éditeurs parisiens et évoquant l'ampleur des recettes qu'il toucherait avec de bonnes ventes (voir 1990, p. 90-92, ou 1992a, p. 17). La plus grande reconnaissance lui vient cependant de ses lecteurs, qui le reconnaissent dans la rue ou dans les transports en commun, ou qui lui écrivent. Il y a là une double implication : outre à reconnaître son rôle d'écrivain, ils l'affirment, le renforcent en l'encourageant ou en lui envoyant des cahiers afin qu'il poursuive son activité (voir 1991a, p. 204). Cette double dynamique de reconnaissance et d'encouragement est à la base de l'existence même du livre, qu'il n'aurait écrit qu'à cause du bouleversement provoqué justement par les lettres qui lui ont été adressées (voir 1991a, p. 9)1․ Certes, d'autres fois, la réaction des lecteurs peut également être négative et prendre presque la forme de l'invective (voir 1989a, p. 95).

\section{Un écrivain entre pudeur et impudeur}

Dans la nouvelle autobiographique «Surtainville, le 13 octobre », qui fait partie du recueil Les Aventures singulières

\footnotetext{
1 Guibert avait annoncé l'intention de ne plus écrire après la parution de $\grave{A}$ l'ami qui ne m'a pas sauvé la vie. Voir Apostrophes du 16 mars 1990.
} 
(Guibert, 1982), le narrateur raconte un voyage entrepris avec le désir de s'isoler dans un endroit paisible pendant quelques jours et d'écrire. Dans le train qui l'emmène vers Cherbourg, il pense à ce qu'il écrira pendant son séjour : «je pensais déjà à ces comptes rendus de la vie d'un voyageur, d'un solitaire» (p. 41). Installé dans sa chambre, qu'il trouve fort décevante, le il sort aussitôt les outils de son travail : «j'ouvris ma valise et en sortis mon stylo-plume, ma bouteille d'encre, deux calepins, du papier, et un volume de Flaubert en Pléiade, que je pris soin de disposer sur la table » (p. 43).

Dans L'Incognito, après avoir établi un état des lieux de sa chambre, il trace une liste des objets qui lui manquent et il indique, entre autres, une chaise et un bureau pour écrire «puisque», remarque-t-il non sans une certaine ironie, «il paraît que je suis écrivain ».

À plusieurs reprises, Guibert raconte ou évoque des séances d'écriture "régulières ", plus ou moins programmées, où lieux, objets, ambiance sont soigneusement choisis et préparés.

D'autres fois, sans doute plus souvent, l'écriture a lieu dans des conditions plus disparates et sur des supports divers : reçus, tickets, bouts de papier. Dans Voyage avec deux enfants, par exemple : «B. me dit avoir retrouvé (une des choses que je crains le plus), dans un livre que je lui avais donné, un de ces mots écrits à la hâte, sur un bout de papier, dans l'autobus ou dans la rue, et que je destine à mon journal » (1992c, p. 50). Guibert explique par la suite qu'il s'agissait d'une « impression» écrite, en tant que telle, pour être inscrite dans son journal intime. La découverte de ses écrits par d'autres est la chose que l'écrivain craint le plus. En effet, un peu plus tard, il 
avoue qu'il écrit, pendant le voyage raconté dans le livre et que le lecteur est en train de parcourir, «à l'écart de T., à son insu, presque en cachette, sans lui rendre compte » (1992c, p. 50-51). La même situation se produit lors d'un trajet en voiture avec Vincent: « [c]es notes dans la voiture, tandis que nous fumons, que je prends sur le petit papier bleu de la banque» (1989b, p. 73-74). Après avoir révélé au lecteur le contenu de ce billet, il nous fait comprendre que l'écriture a lieu en cachette et qu'elle n'est nullement partagée avec son compagnon de route, puisque Guibert précise : "Vincent détache son regard de la route pour tenter de voir ce que j'écris sur le ticket » (1989b, p. 74). Parfois, selon des modalités identiques, l'auteur évoque son besoin d'écrire à l'aéroport; pendant qu'il attend son vol dans la salle d'embarquement, il n'arrive pas à résister à la tentation de prendre la plume et il cède : «tanné par les mots, j'ai fini par sortir mon carnet ». Il éprouve cependant un certain ennui, lorsqu'«un homme est venu s'assoir à coté de [lui] comme pour lire par-dessus [s]on épaule» (1992c, p. 71) et il réfléchit, par conséquent, aux caractéristiques et à l'aspect de son écriture, qui « a l'immense désavantage d'être trop lisible, il va falloir que je la rende la moins lisible possible » (1992c, p. 71-72). Guibert manifeste, en somme, une sorte de retenue, de pudeur à l'égard de ses manuscrits et de leur contenu, avec l'inquiétude constante que ceux-ci tombent entre de mauvaises mains. Non seulement envers les inconnus mais aussi avec ses proches, l'écrivain manifeste la peur que l'on lise ses papiers, ses notes, ses brouillons, son œuvre au stade embryonnaire. Par exemple, au sujet de la cohabitation avec Jules et Berthe, les amis avec qui il se lie intimement, il observe: «je tremblais chaque jour de peur que Berthe ne mette son nez dans ce manuscrit que je laissais sur le bureau» (1990, p. 146); le 
narrateur est de même très contrarié quand il s'aperçoit que son ami Gustave a lu, à son insu, son journal intime, source inépuisable de matière littéraire :

En arrivant dans la pièce où Gustave se trouvait j'ai entendu claquer un cahier, il venait de refermer mon journal, que j'avais laissé en confiance avec mon manuscrit en cours sur la table de la salle à manger. Il m'a aussitôt dit : "C'est la première fois." Ce n'est pas tant le fait que Gustave ait lu mon journal qui est grave, que le fait que je l'y ai surpris. (1989a, p. 217)

Dans un autre cas, en prévision d'une opération chirurgicale, l'écrivain interpelle, par précaution, l'ami H. G., comme il nous le relate dans un fragment de son journal d'hospitalisation Cytomégalovirus : " [j]'ai confié le premier carnet à H. G., dans une enveloppe fermée, sans lui dire ce que c'était, parce que demain je descends au bloc opératoire à neuf heures et que j'avais peur qu'on me le dérobe, dans ma chambre, ou même dans ma poche » (1992d, p. 55). Non seulement l'écrivain craint le vol, mais il empêche également ses proches de lire ses papiers personnels: le carnet est en effet fermé dans une enveloppe et H. G. n'est pas au courant de ce qu'elle contient. Lors de la même hospitalisation, Guibert est conduit au service radiologie pour des examens et la même préoccupation, par ailleurs la seule, l'envahit au moment de quitter la chambre : «j'ai d'abord caché mon carnet sous l'oreiller et puis je me suis dit que c'était une très mauvaise idée, il rentrait dans la poche de ma chemise » (1992d, p. 71). Cette peur de la disparition, du vol ou de l'intrusion est largement répandue dans l'œuvre et frôle parfois la paranoïa. Ainsi, à l'occasion d'un voyage à Moscou effectué dans des conditions de précarité physique mais aussi d'instabilité sociale et politique dues au régime soviétique, l'écrivain trace l'inventaire des objets qu'il emmène 
avec lui. Contrairement à ce à quoi on s'attendrait, il laisse à Paris les matériaux pour écrire: "je n'avais emporté comme livre que l'œuvre autobiographique de Strindberg réunie en volume au Mercure de France. Rien pour écrire moi-même, j'avais trop peur que l'inspectrice bilingue, déguisée en femme de chambre, de l'hôtel Rossia, ne recopie au fur et à mesure mon journal » (1992a, p. 139). Il résume ainsi son séjour moscovite, établissant un parallèle entre l'impossibilité d'écrire et la pauvreté des conditions : « [s]ix jours sans écriture, nourri au yaourt et au potage lyophilisé réchauffé avec une résistance dans un verre à dents infesté de bactéries » (1992a, p. 139).

\section{Les « manuscrits corporels »}

Si Guibert fonde son œuvre sur l'écriture du corps, et si corps et écriture se confondent constamment, à tel point que la critique a, dès le début, parlé d'«écriture corporelle», où le corps devient un véritable «principe générateur de l'œuvre» (Sarkonak, p. 7-9), l'écriture sur l'écriture s'inscrit dans cette réflexion sur le corps, sur son propre corps et sur le corps d'autrui : corps imaginé, corps fragmenté, corps rêvé, corps infecté, corps jouissant, corps mourant. Pour Guibert, en effet « [l] corps est toujours une obsession fictionnelle » (Boulé, p. 104) et l'on peut parler d'une constante « identification entre corps et écriture » (p.105). L'écriture n'est cependant pas à envisager uniquement en termes abstraits : l'attention portée à l'objet-manuscrit prend une allure de plus en plus radicale, aboutissant à une sorte de cristallisation de cet objet, de prolongement du sujet qui est de plus en plus identifié à son manuscrit. Le corps ne s'identifie pas seulement à l'écriture en tant que telle, mais aussi au support, à l'objet tangible. Tandis 
que le corps de l'écrivain s'affaiblit, disparaît, son manuscrit demeure, prend sa place, mais il est sujet aux intempéries du monde et doit être préservé. Le corps et le manuscrit participent de concert à l'affirmation d'une seule identité et d'une seule représentation.

Écrire pour ne pas oublier, écrire pour fixer l'impression, l'image, le sentiment qui, comme un éclair, apparaît et passe dans la tête. Écrire, finalement, pour profiter du temps qui reste, dans la hantise imposée par l'avancement de la maladie, l'affaiblissement du corps, la menace de la cécité (voir Guibert, 1990, p. 160). L'écriture guibertienne devient corporelle aussi dans ce sens où elle est soumise au corps, à son changement, à sa force et à sa faiblesse, à son potentiel et à son impuissance :

J'avais écrit bien assez de livres comme ça : il y en avait treize écrits, treize publiés et disponibles sans compter ceux que j'avais discrètement rayés de "la liste du même auteur". J'ai tellement écrit depuis que j'ai quinze ans! Quand je regarde les plis de dossiers entassés dans les placards, je n'en crois pas mes yeux, je suis effaré, je n'arrive plus à comprendre comment une masse d'écriture si étendue a pu sortir de mon pauvre corps et de mes pensées pitoyables. (1991a, p. 194-195)

Regardant ses manuscrits accumulés au fil du temps dans ses placards, l'écrivain exprime son incrédulité comme si, se regardant dans un miroir, il ne reconnaissait pas sa propre image, l'image de soi que ses manuscrits et leur épaisseur lui renvoient. L'écriture n'est donc pas une activité uniquement intellectuelle, mais elle relève aussi de la vigueur physique de l'écrivain, comme si la production matérielle des textes prenait l'intensité pathétique d'un accouchement, d'une séparation de son corps, de sa fibre. 
À l'instar de n'importe quel autre métier, l'écriture fatigue le corps, le plie à ses rythmes et à ses contraintes, et ce, à plus forte raison, au fur et à mesure que la maladie avance : " [d]ans l'état où je suis j'ai besoin d'un serviteur, un jeune homme vigoureux qui me conduise, m'habille, me lave, masse mon dos de plus en plus endolori par l'écriture » (1991a, p. 178).

Cette implication du corps et la confusion entre physiologie et production textuelle sont encore présentes dans l'idée que l'effet de l'assomption d'un médicament suscite comme seul effet la production d'un texte: «je me sens toujours aussi mal, et je suis en attente de la délivrance de ce médicament, que je prends en réalité depuis cinq jours, sans sentir d'autre effet que la production de ce récit» (1991, p. 25) ; ou encore :« [c]'est le DDI du danseur mort, avec le Prozac, qui écrit mon livre »; et il ajoute: «ça me fait quelque chose de savoir que ce sont des substances chimiques qui écrivent mon livre » (1991, p. 99). À plusieurs reprises, l'idée revient d'une écriture qui, comme un fluide, une sécrétion, comme le sang infecté, envahit le corps de celui qui entre en contact avec l'écrivain en lisant, que ce soit un journal ou un texte littéraire : Guibert, comme tout lecteur, ne peut se préserver de cette contagion. À ce propos, le parallèle entre le virus et l'influence littéraire est particulièrement significatif avec Thomas Bernhard: "parallèlement donc au virus HIV la métastase bernhardienne s'est propagée à la vitesse grand $V$ dans mes tissus et mes réflexes vitaux d'écriture, elle la phagocyte, elle l'absorbe, la captive, en détruit tout naturel et toute personnalité pour étendre sur elle sa domination rageuse » (1990, p. 232). 
L'idée de l'investissement du corps dans l'écriture est renforcée par un discours où les écrits, les manuscrits deviennent des véritables prolongements du corps, presque des fétiches de l'écrivain qui les a produits, quand il disparaît. En particulier, Guibert rapporte l'attention de Muzil-Foucault pour ses propres manuscrits et les efforts presque obsessionnels de ce dernier afin d'assurer leur destruction après sa mort. Avant son départ pour un voyage en Andalousie, il convoque Guibert et, en lui montrant de grosses chemises posées sur son bureau, il lui donne des consignes très claires: " "[c]e sont mes manuscrits, s'il m'arrivait quoi que ce soit durant ce voyage, je te prie de venir ici et de les détruire tous les deux, il n'y a que toi à qui je peux demander ça, et je compte sur ta parole" » (Guibert, 1990, p. 38). Guibert se dit incapable d'accomplir une telle tâche, comme si l'objet-manuscrit, sacralisé, était hors de la volonté et du pouvoir de celui qui l'a créé et qui en détient les droits et la propriété. Après la mort du philosophe, son compagnon Stéphane négocie avec la famille l'obtention de l'appartement «avec les manuscrits qui s'y trouvaient» (Guibert, 1990, p. 116), même s'il ignore qu'ils ont été pour la plupart détruits par Foucault lui-même avant sa disparition. Malade, le philosophe veut empêcher toute publication posthume, parfaitement conscient des risques de manipulation et de spéculation qu'une publication non surveillée comporterait. Comme s'ils étaient partie intégrante de lui, de son identité, de son physique, ses manuscrits disparaissent avec lui. Les manuscrits sauvés de la destruction se cristallisent après la mort de leur auteur ainsi que les autres objets et certains lieux, devenant «intouchables»; Stéphane invite Guibert dans l'appartement et lui «montr[e], empilés dans un placard, les manuscrits, toutes les ébauches et les brouillons du livre infini 
qui n'avaient pas été déchirés », lui demandant également de prendre des photos, comme pour fixer à jamais une dernière image de l'ami mort (Guibert, 1990, p. 124-125).

L'Homme au chapeau rouge se termine par un avertissement métatextuel: "[i]ci se trouvaient cinquante pages de mon manuscrit qui ont été perdues. C'était le récit de mon voyage en Afrique » (Guibert, 1992a, p. 165). À la fin de l'histoire la plus romanesque du cycle du sida, l'auteur insère cet épisode, cette dernière aventure scripturale, qui souligne la nature précaire de l'écriture, liée à sa matérialité toujours éphémère, presque à la merci du hasard :

j'ai eu un curieux pressentiment en regardant partir mon bagage sur le tapis roulant de l'enregistrement. Il était déjà en route quand je l'ai rattrapé pour le rouvrir, j'ai eu la présence d'esprit de récupérer le cahier noir sur lequel je tiens mon journal depuis quinze ans, mais j'ai laissé mon manuscrit au fond du sac. J'ai compris à Roissy que je ne remettrais jamais la main dessus. (1992a, p. 156-166)

Inutile pour l'écrivain d'essayer de réécrire ce qui a été perdu dans ces « cinquante pages égarées, qui sont maintenant Dieu sait où », puisqu' " une fois que les choses sont écrites elles sont pour moi comme effacées »(1992a, p.166). Dans la tête de l'auteur, les mêmes dynamiques que l'on trouve dans les manuscrits prennent place : la trace écrite est aussitôt effacée, écriture et rature cohabitent, occupent le même espace. On revient donc à l'idée d'une écriture liée à l'instant, à la circonstance, une écriture qui prend des clichés d'émotions, de sentiments, de désirs.

On assiste également à l'inverse. L'écriture oubliée et que le hasard fait resurgir est tout à fait capable d'évoquer des souvenirs lointains et de rappeler des émotions, des 
impressions déjà perdues. Au cours d'un déménagement, Guibert, mettant de l'ordre dans ses dossiers, retrouve des vieux cahiers oubliés :

[j]'y ai retrouvé, surtout dans ces cahiers, des choses que j'avais écrites quand j'étais très jeune, que je n'avais jamais mises au propre, et que j'avais souvent complètement oubliées, comme si elles avaient été écrites par un autre, un être plus rare et plus pur que moi, ce jeune Guibert qui me faisait le cadeau, par ces textes, de me faire croire qu'il était resté moi-même, que nous n'étions qu'une seule personne. (1991, p. 196)

La redécouverte de textes déjà oubliés s'accompagne d'une plus vaste réflexion sur l'écriture et sur son potentiel, qui se traduit par des sentiments contradictoires: "la redécouverte de ces textes parfois m'enchantait, parfois m'horripilait» (1991, p. 196). Dans la même page, l'auteur poursuit, parlant de son travail d'écrivain : «je m'étais mis à travailler dans ces cahiers d'adolescence, je n'y touchais pas, je me contentais de choisir et de taper à la machine». Il insiste, en outre, sur la réappropriation, du point de vue matériel aussi, de ces écrits : «je sentais bien en même temps qu'après leur repossession, cette attribution que je me réservais puisque je les tenais matériellement comme quelqu'un qui en aurait hérité, je ne pourrais plus écrire comme avant. Ces textes me modifiaient comme écrivain » (1991, p. 197; c'est moi qui souligne). Il faut mettre l'accent, ici, sur le caractère tout matériel de cette repossession. C'est la détention physique des textes, conçus en tant que supports, manuscrits, qui permet à l'écrivain de reconnaître, pour ainsi dire, sa paternité. Le jeune Guibert, auteur des textes, les laisse en héritage à l'écrivain adulte, lequel ressent le droit d'en prendre possession avant tout en vertu de leur statut d'objets. Ceux-ci, contenant des textes, ont 
le pouvoir de le modifier, d'influencer son identité d'écrivain, son écriture.

Ce qui en dérive est une sorte de désir d'imitation de sa propre écriture d'autrefois, de réappropriation de ce qui a été, du corps et d'autant de circonstances oubliées. Claire aux yeux de l'auteur est en revanche l'impossibilité de "réatteindre », de poursuivre le même type d'écriture. Les textes sont en effet placés sous une sorte de protection impénétrable, exercée par l'écriture même :

ces couches de sédimentation de l'écriture accomplie et relativement oubliée, cette mémoire inconsciente, parfois désastreuse parfois salutaire, de l'écriture exercée de l'artisan, de l'instrumentiste dirait Bernhard, qui revenait à la charge pour vous empêcher d'écrire comme un autre que soi-même, parce que ce soi-même aurait été scellé dans l'écriture. (1991, p. 197)

L'écriture se protège elle-même et empêche l'écrivain d'écrire comme un autre. Guibert déclare par là même l'importance des textes de jeunesse apparemment «obscurs, obscènes, aux limites de la lisibilité » (1991, p. 197), mais revêtant la plus haute importance dans l'ensemble de la production littéraire et artistique guibertienne, et capables de modifier la perception qu'il a de lui-même en tant qu'écrivain.

Guibert nous ouvre aussi les portes d'un autre univers, qui est celui de la création, de la conception mentale de l'œuvre littéraire. Resté seul à Rome pendant les vacances de Noël, il raconte: «[j]'entrevois l'architecture de ce nouveau livre que j'ai retenu en moi toutes ces dernières semaines mais j'en ignore le déroulement de bout en bout, je peux en imaginer plusieurs fins » $(1990$, p. 10-11). L'acte imaginatif prend de plus en plus l'allure de l'acte d'écrire, comme si l'auteur était en train d'esquisser des plans de son œuvre : 
En m'endormant je repense à ce que j'ai écrit pendant la journée, certaines phrases reviennent et m'apparaissent incomplètes, une description pourrait être encore plus vraie, plus précise, plus économe, il y manque tel mot, j'hésite à me relever pour l'ajouter, j'ai quand même du mal à descendre du lit, à chercher dans le noir à tâtons la lampe de poche à travers la moustiquaire, ramper sur le côté au bord du matelas comme me l'a enseigné le masseur, et laisser tomber doucement mes jambes, jusqu'à ce que mes pieds rencontrent la pierre nue, allumer une bougie, chercher la bonne page dans le manuscrit, perfectionner par un ajout ou une biffure la phrase en question. Sinon, retrouverai-je demain le mot qui manquait? Non. (1991, p. 144)

Une fois fixée sur la page, l'écriture revient à l'auteur, le hante, continue à évoluer dans sa tête.

À travers les quelques exemples que nous venons d'évoquer, nous essayons de donner un échantillon du caractère autoréflexif que Guibert confère, à divers degrés, à ses textes. L'auteur ne se limite pas à nous montrer son travail, son bureau, les feuilles qu'il écrit, mais il approfondit cette investigation jusqu'à compléter une interrogation sur la nature de l'objet-manuscrit et sur la consistance de l'écriture où réside, au fond, le mystère de la création, de l'œuvre littéraire.

S'inscrivant de façon singulière dans le projet de dévoilement de soi, l'écriture, montrée ou cachée, revient toujours, resurgit du néant, de l'oubli, permet au corps mourant de subsister, au corps vivant de retrouver ses dimensions d'autrefois. Les supports, les matériaux, les modalités de travail acquièrent la valeur de prolongement du corps et de l'identité au centre de l'œuvre guibertienne, et ils nous sont montrés par le biais des mêmes modalités, des mêmes effets de clair-obscur, de voilement et dévoilement qui caractérisent tant d'images et d'autoportraits, aussi bien photographiques que littéraires, de l'auteur. À ce propos, l'accent doit tout particulièrement être 
mis sur le rôle que la dimension matérielle de l'écriture acquiert dans l'expérience littéraire de Guibert. Les gestes, les objets, les manuscrits, dans leur épaisseur tangible, permettent à l'auteur de tracer ou de photographier son identité scripturaire. Consubstantiels au corps de l'écrivain, ils sont sujets à la même précarité, aux mêmes risques, mais en même temps, ils sont capables de défier le temps en devenant des substituts de leur auteur après la disparition de celui-ci. Quand l'écrivain cache ou met à l'abri ses écrits et son écriture, c'est son corps, son identité qu'il voile. Par contre, le geste d'ouverture de son atelier ou de son carnet relève du projet de dévoilement inscrit dans le manifeste lancé en 1977 (voir Guibert, 1977). L'écrivain affirmé, à la recherche de ses jambes et ses bras d'enfant (voir 1990, p. 284), ne cesse de réfléchir à propos de ses modes de travail, de son écriture et de revenir aux objets et aux actes de sa naissance littéraire, là où, justement, tout a commencé avec le geste primordial, toujours répété : «aux interrogations je rends des feuilles blanches. Et dans les marges du cahier de textes, toujours, je dessine un visage maigre, en forme de lame de couteau, toujours le même : je crois ne pas le connaître, et l'inventer » $(1986$, p. 84).

\section{Bibliographie}

BoulÉ, Jean-Pierre (2001), Hervé Guibert: l'entreprise de l'écriture du moi, Paris, L'Harmattan.

BRUNET, Mathieu. (2010), «Autoréflexivités paradoxales : lire ou ne pas lire?», dans Jan Herman et al. (dir.), L'Assiette des 
fictions. Enquête sur l'autoréflexivité romanesque, Louvain, Peeters, p. 253-263.

GEnon, Arnaud. (2007), Hervé Guibert : vers une esthétique postmoderne, Paris, L'Harmattan.

GuiberT, Hervé. (1977), La Mort propagande, Paris, Régine Déforges.

—. (1982), Les Aventures singulières, Paris, Minuit.

—. (1986), Mes Parents, Paris, Gallimard.

—. (1989a), L'Incognito, Paris, Gallimard.

-. (1989b), Fou de Vincent, Paris, Minuit.

—. (1990), À l'ami qui ne m'a pas sauvé la vie, Paris, Gallimard.

—. (1991), Le Protocole compassionnel, Paris, Gallimard.

—. (1992a), L'Homme au chapeau rouge, Paris, Gallimard.

—. (1992b), Cytomégalovirus, Paris, Seuil.

-. (1992c), Voyage avec deux enfants, Paris, Gallimard.

—. (1992d), Cytomégalovirus, Paris, Seuil.

HERman, Jan et al. (dir.). (2010), L'Assiette des fictions. Enquête sur l'autoréflexivité romanesque, Louvain, Peeters.

LEPALUDIER, Laurent. (2002), «Fonctionnement de la métatextualité: procédés métatextuels et processus cognitifs », dans L. Lepaludier (dir.), Métatextualité et métafiction. Théories et analyses, Rennes, Presses universitaires de Rennes, p. 25-38.

PUJADE, Robert. (2008), Hervé Guibert. Une leçon de photographie, Lyon, Université Claude Bernard Lyon 1. 
SARKONAK, Ralph. (1997), « Du para- au métatexte. À l'ami qui ne m'a pas sauvé la vie », dans Ralph Sarkonak (dir.), Le Corps textuel d'Hervé Guibert, Revue des Lettres Modernes.

WESSLER, Éric. (2009), La Littérature face à elle-même. L'écriture spéculaire de Samuel Beckett, Amsterdam, Rodopi.

\title{
Résumé
}

Faisant de lui-même le personnage principal de son œuvre, Hervé Guibert nous parle nécessairement de son travail d'écrivain. À travers ses récits, il dessine l'image d'un écrivain polymorphe, qui prend une double attitude par rapport à son métier. Si, d'un côté, l'acte de l'écriture et les manuscrits sont montrés, de l'autre, il ne cesse d'accuser sa retenue et à manifester une sorte de pudeur. À la recherche d'une identité scripturaire, l'auteur tend à assimiler ses manuscrits à son corps, à sa fibre, dans un projet global de dévoilement de soi.

\begin{abstract}
Being the main character of his own books, Hervé Guibert writes about his life as a writer. Through his texts he draws his portrait as a polymorphous artist, taking a double attitude towards his activity. On the one hand the act of writing, as well as drafts and manuscripts are shown, but on the other hand he constantly tries to protect and conceal them from sight. Looking for his own scriptural identity, the author tends to identify his manuscripts with his body, so that papers, drafts, manuscripts come to acquire a strong corporeal dimension in a wider project of representation of the self.
\end{abstract}

